\title{
ORIGINAL
}

\section{EVALUACIÓN Y MEJORA DE LA CALIDAD \\ DE LOS DOCUMENTOS DE CONSENTIMIENTO INFORMADO EN NUEVE HOSPITALES DEL SERVICIO MURCIANO DE SALUD}

\author{
José Eduardo Calle-Urra (1), Pedro Parra-Hidalgo (1), Pedro Saturno-Hernández (2), Yadira Fonseca- \\ Miranda (1) y Ma Jesús Martínez-Martínez (1).
}

(1) Consejería de Sanidad y Política Social de Murcia. Programa EMCA.

(2) Universidad de Murcia. Programa EMCA.

Los autores declaran no tener ningún conflicto de intereses

\section{RESUMEN}

Fundamentos: Los documentos de consentimiento informado tienen gran importancia en el proceso de información médica. El objetivo de este trabajo fue evaluar los cambios producidos en los niveles de calidad formal de estos documentos como consecuencia de las medidas correctoras puestas en marcha tras la primera evaluación.

Métodos: Ciclo completo de evaluación y mejora de la calidad con 4 evaluaciones entre los años 2007 y 2013 . Se valoraron 19 criterios en 3.045 documentos pertenecientes a 9 hospitales del Servicio Murciano de Salud. En la primera evaluación se estudiaron todos los documentos y en las restantes una muestra aleatoria. A partir de los resultados de la primera evaluación se pusieron en marcha medidas correctoras. Para valorar su efectividad se calculó el porcentaje de incumplimiento de los criterios y el porcentaje de mejora relativa entre la primera y la última evaluación Para valorar la variabilidad se obtuvo la razón de variación entre hospitales en el promedio de defectos por documento.

Resultados: La mejora alcanzada fue del 40,8\%. Esta oscilaba entre e $94 \%$ en el nombre del centro y el $17,6 \%$ en las consecuencias relevantes, siendo las diferencias encontradas en 16 de los 19 criterios estadísticamente significativas. El promedio de defectos por documento descendió de 7,6 a 4,5 con una razón de variación entre hospitales de 8,33.

Conclusiones: La mejora alcanzada en la calidad de los documentos de consentimiento informado está relacionada con la efectividad de las medidas correctoras puestas en marcha.

Palabras clave: Formularios de consentimiento. Normas. Estudios de evaluación. Mejoramiento de la calidad. Hospitales. Control de calidad.

Correspondencia

José Eduardo Calle Urra

Consejería de Sanidad y Política Social de Murcia

C/ Pinares $n^{\circ} 6,2^{\mathrm{a}}$ planta

30.001-Murcia

josee.calle@carm.es

\section{ABSTRACT \\ Evaluation and Improvement of the Informed Consent Documents in 9 Hospitals, Spain}

Background: Informed consent forms are very important in the process of medical information. The aim of this study was to assess the changes in the formal quality levels of these documents, as a result of corrective measures implemented after the first evaluation.

Methods: Complete cycle of evaluation and quality improvement, with 4 assessments between the years 2007 and 2013. They were assessed 19 criteria in 3,045 documents belonging to 9 hospitals of Murcian Health Service. In the first evaluation the universe was studied and in the others a random sample. From the results of the first assessment corrective measures were implemented. To evaluate its effectiveness the percentage of nonobservances per criteria was obtained, calculating the percentage of relative improvement between the first and the last evaluation. To assess the variability among hospitals the high-low ratio in the average number of defects per document was obtained.

Results: The percent of improvement achieved was $40.8 \%$. This ranged from $94 \%$ in the name of the centre to $17.6 \%$ in the relevant consequences, being statistically significant in 16 of the 19 criteria. The average number of defects per document decreased from 7.6 to 4.5 , with a high-low ratio among hospitals of 8.33 .

Conclusions: The improvement achieved in the quality of informed consent documents is related to the effectiveness of corrective measures implemented.

Key words: Consent forms. Standards. Evaluation studies. Quality improvement. Hospitals. Quality control.

DOI: 


\section{INTRODUCCIÓN}

La relación más importante en la asistencia sanitaria es la del médico con el paciente. Esta se ha desarrollado durante mucho tiempo mediante un modelo según el cual el profesional, adoptando un papel paternalista, decidía en nombre del paciente según su criterio exclusivo. Durante los últimos años este modelo ha entrado en crisis y ha sido sustituido por otro basado en el principio de autonomía, según el cual el paciente, para que tenga lugar una actuación que afecta a su salud, debe dar su consentimiento, después de recibir la información adecuada ${ }^{1}$. El documento del consentimiento informado (DCI) es el soporte documental de este proceso y trata de garantizar que la información más relevante ha sido ofrecida por el médico y recibida por el paciente ${ }^{1}$. Es una importante fuente de información que complementa a la obtenida de forma oral y permite completar el proceso de toma de decisión.

La teoría del consentimiento informado se gestó a finales del siglo XVIII en Estados Unidos, pero su verdadero desarrollo se produjo en el contexto del movimiento de reivindicación de los derechos civiles que, iniciándose a finales de la II Guerra Mundial, tuvo su auge durante los años sesenta y setenta del siglo $\mathrm{XX}^{2}$. El concepto de consentimiento informado fue utilizado por primera vez en 1957, en el Estado de California, en el caso de Martín Salgo contra Leland Stanford Jr Universitary Board of Trustees ${ }^{1,3}$.

En España, ya en 1972, el artículo 148.4 del Reglamento General para el Régimen, Gobierno y Servicio de las Instituciones Sanitarias de la Seguridad Social ${ }^{4}$, establecía el derecho de los enfermos a autorizar las intervenciones quirúrgicas y las actuaciones terapéuticas que implicaran riesgo notorio previsible, así como a ser advertidos de su gravedad, pero no fue hasta 1986 cuando el artículo 10 de la Ley General de Sanidad estableció el derecho de los usuarios del sistema sanitario a la información completa y continuada, verbal y escrita, sobre su proceso, a la libre elección entre las opciones que le presente el responsable médico, y a la necesidad de obtener el previo consentimiento escrito para la realización de cualquier intervención, excepto en los casos de riesgo para la salud pública, incapacidad para tomar decisiones o cuando la urgencia no permita demoras 5 .

Posteriormente la Ley 41/2002 de Autonomía del Paciente estableció la obligación de obtenerlo por escrito ante intervenciones quirúrgicas, procedimientos diagnósticos y terapéuticos invasores y siempre que se aplicaran procedimientos que supusieran riesgos o inconvenientes con repercusiones negativas sobre la salud del paciente ${ }^{6}$.

El consentimiento informado es sobre todo verbal, pero la disponibilidad de documentos de consentimiento informado estandarizados en entornos clínicos con sobrecarga asistencial los convierten muchas veces en la principal, si no la única, fuente de información del paciente, por lo que es importante que estos documentos sean de calidad ${ }^{7}$.

En un estudio anterior se definieron criterios de calidad formal explícitos, se evaluó la totalidad de los DCI utilizados en una red de hospitales públicos y se realizó una propuesta de medidas para la mejora ${ }^{8}$.

El objetivo de este trabajo fue evaluar los cambios producidos en los niveles de calidad formal de los documentos de consentimiento informado como consecuencia de las medidas correctoras puestas en marcha tras la primera evaluación.

\section{MATERIAL Y MÉTODO}

El Servicio Murciano de Salud (SMS) es el organismo público encargado de la provisión de servicios sanitarios en la Comunidad Autónoma de la Región de Murcia que en 2007, año al que correspondían los DCI de la primera evaluación, contaba con una población de referencia de 1.392.117 habitantes y 9 hospitales públicos generales, con un número de camas entre 97 y 877 según el hospital. 
Tipo de estudio. Ciclo completo de evaluación y mejora de la calidad (estudio cuasi-experimental) con cuatro evaluaciones externas.

Dimensión de calidad evaluada. Calidad científico-técnica y atención centrada en el paciente.

Unidades de estudio. Documentos de consentimiento informado que, en el momento del estudio, estuviesen siendo utilizados en los hospitales generales del SMS, excluyendo los relacionados con intervenciones de carácter experimental. Se excluyeron también los documentos que no estuviesen redactados en español, los utilizados para fines distintos a la petición del consentimiento, los repetidos, los relacionados con técnicas que no eran realizadas por el centro, los que contuviesen información para más de un procedimiento o intervención (DCI múltiples), los de carácter genérico y cuando no coincidiera el nombre del procedimiento con el de la intervención para la que finalmente se otorgaba la autorización o con el contenido del documento.

Universo o marco muestral. Total de DCI válidos utilizados por los hospitales en los años 2007, 2010, 2011 y 2013. El número global de DCI válidos del universo fue de 9.540 .

Tamaño muestral, tipo de muestreo y mecanismo de sustitución de las unidades de estudio. En total se evaluaron 3.045 documentos. En la primera evaluación se estudió la totalidad del universo (1.425 DCI tras aplicar los criterios de exclusión a los 1.676 que habían sido remitidos por los hospitales). En la segunda, tercera y cuarta evaluaciones se estudiaron 60 documentos en cada centro (540 para el total del SMS), obtenidos mediante muestreo aleatorio estratificado no proporcional por hospital. El tamaño muestral utilizado para el total del SMS permitió detectar como significativas diferencias iguales o superiores a 0,05 en todos los $\operatorname{casos}^{9}$.

Se estableció un mecanismo de sustitución consistente en que en el caso de que no pudie- se ser valorado el DCI seleccionado se extraía el siguiente, también de manera aleatoria.

Variables de estudio: diseño de los criterios de calidad. Para la evaluación de los documentos se utilizaron los 19 criterios de calidad que aparecen en el anexo 1, junto a sus aclaraciones y excepciones.

La elaboración de los criterios de calidad se llevó a cabo de acuerdo a la metodología recomendada para ello y que ya ha sido descrita en un artículo previo ${ }^{8}$.

Los criterios hacían referencia, en todos los casos, a la presencia o ausencia del aspecto evaluado, no valorándose si el contenido del mismo era adecuado o no.

Medidas de intervención propuestas tras la primera evaluación. Una vez realizado el análisis de los datos de la primera evaluación, se remitió el informe con los resultados a los responsables del estudio en los hospitales, elaborándose las siguientes recomendaciones:

- Identificación de los procedimientos que tienen que disponer de DCI escrito dentro de cada especialidad.

- Elaboración de todos los documentos conforme a los criterios de calidad propuestos.

-Puesta en marcha de un sistema de registro y acreditación de los documentos utilizados en el centro.

-Utilización de documentos que contengan epígrafes que separen cada uno de los apartados de información contenidos en los mismos.

- Reducción al máximo del uso de siglas. Si fuese imprescindible utilizar alguna, aclarar su significado entre paréntesis la primera vez que aparezca en el DCI.

- Difusión de los datos y realización de actividades de formación entre los profesio- 
nales sanitarios de los centros, con el objetivo de concienciar a los mismos acerca de la importancia y ventajas de disponer de DCI de calidad como paso previo para su mejora.

De forma paralela, con independencia de las actividades de formación realizadas en cada uno de los hospitales, se incluyó la información de este proyecto en los cursos sobre Evaluación y Mejora de la Calidad impartidos por el Programa EMCA (Evaluación y Mejora de la Calidad Asistencial) a los profesionales sanitarios del SMS.

Pasados diez meses desde el envío de los resultados de la primera evaluación se remitió otro informe a todos los responsables del estudio, con el fin de completar el análisis realizado en el anterior. Este segundo informe se acompañó de una carta en la que se recordaban las principales medidas correctoras que debían ser puestas en marcha por los centros.

Se diseño un modelo de DCI por parte de un facultativo de la Unidad de Medicina Judicial y Ética Médica de uno de los hospitales. Tras ser revisado y aprobado por el Comité de Ética Asistencial y Dirección Médica del centro, fue remitido al investigador principal del estudio para su evaluación con los criterios de calidad propuestos. Dado que el modelo cumplía con todos los requisitos, se recomendó su utilización en el rediseño de los documentos hospitalarios.

Además, en los contratos de gestión del SMS de 2010, 2011 y 2013, se incluyó como objetivo la realización de reevaluaciones de los criterios de calidad, con el objeto de valorar el grado de efectividad de las medidas correctoras. Una vez realizado el análisis de los datos se remitió un informe con los resultados a todos los responsables del estudio en los hospitales.

Análisis estadístico. Aunque los criterios de calidad ya habían demostrado su fiabilidad en la primera valoración ${ }^{8}$, como el resto de las evaluaciones fueron realizadas por tres profesionales sanitarias (1 psicóloga y 2 enfer- meras) que eran distintas a la enfermera que participó en la primera valoración, se procedió de nuevo al entrenamiento en el manejo de los criterios y al cálculo del índice kappa de concordancia antes de dar comienzo al trabajo de campo de reevaluación de los DCI.

Para cada hospital se calculó el porcentaje de DCI sin epígrafes en cada uno de los ítems de información de cada una de las evaluaciones así como la diferencia absoluta y relativa entre la cuarta y la primera valoración. La diferencia absoluta se calculó restando al porcentaje de DCI sin epígrafes en la cuarta evaluación el porcentaje obtenido en la primera, y la diferencia relativa se obtuvo dividiendo la diferencia absoluta entre el porcentaje de documentos sin epígrafes de la primera valoración.

Para valorar la efectividad de las medidas correctoras sobre el nivel de cumplimiento de los criterios de calidad se calcularon los siguientes indicadores:

1.- Promedio de defectos por DCI en cada una de las evaluaciones desagregado por hospital y diferencia absoluta y relativa entre la cuarta y la primera valoración. La diferencia absoluta se calculó restando al promedio de defectos por documento de la cuarta evaluación el promedio obtenido en la primera, y la diferencia relativa dividiendo la diferencia absoluta entre el promedio de incumplimientos por DCI de la primera evaluación.

Para calcular la variabilidad en la calidad global de los documentos en la cuarta evaluación se obtuvo la razón de variación entre centros del promedio de defectos por DCI, siendo el cociente entre el valor máximo y el mínimo obtenido por los hospitales.

2.- Porcentaje de incumplimiento de los criterios en cada una de las evaluaciones (estimación puntual e intervalo de confianza del $95 \%$ ), para el total de hospitales y por tipo de criterio, y diferencia absoluta y relativa entre la cuarta y la primera evaluación. La diferencia absoluta se calculó restando al porcentaje 
de incumplimiento de la cuarta evaluación el porcentaje obtenido en la primera, y la diferencia relativa dividiendo la diferencia $a b-$ soluta entre el porcentaje de incumplimiento de la primera evaluación.

Se consideró que las diferencias eran estadísticamente significativas cuando el intervalo de confianza de la cuarta evaluación no incluía la estimación puntual de incumplimiento de la primera, dado que esta fue obtenida sobre la totalidad del universo de DCI válidos remitidos desde los hospitales $^{10}$.

En las reevaluaciones, el cálculo de los porcentajes de incumplimiento de los criterios para el total de hospitales se realizó a partir de las fórmulas para muestras estratificadas no proporcionales, es decir, ponderando por el universo de DCI válidos de cada centro $^{11}$.

Los criterios se clasificaron, en función de su contenido, en los mismos grupos que ya fueron utilizados en la primera evaluación ${ }^{8}$. Se trata de tres grupos que hacen referencia a los "datos sobre fecha y lugar del consentimiento y datos administrativos del centro, médico, paciente y representante legal", a los "datos del procedimiento a realizar" y, finalmente, a los "datos sobre comprensión del consentimiento, posibilidad de revocación, autorización para la realización y entrega de copia".

La importancia de esta clasificación estriba en que el uso por los centros del modelo corporativo de consentimiento informado que fue propuesto en las medidas correctoras les habría permitido reducir a cero el nivel de defectos en los criterios de los grupos 1 y 3 y del criterio 12 (riesgos personalizados) del grupo 2.

Para poder visualizar mejor las mejoras alcanzadas entre la primera y la cuarta evaluación, así como cuáles eran los criterios que acumulaban el mayor porcentaje de defectos, se diseñó un gráfico de Pareto antes- después para el global del SMS. Este gráfico se obtiene a partir del número total de incumplimientos de cada criterio en cada una de las evaluaciones, que es utilizado para calcular su frecuencia relativa y la frecuencia acumulada de defectos ${ }^{9}$.

Todos los análisis se llevaron a cabo a partir de la última versión disponible del paquete estadístico SPSS en el momento realizar cada evaluación.

\section{RESULTADOS}

En el análisis de concordancia realizado antes de la segunda evaluación 16 de los 19 criterios diseñados obtuvieron niveles kappa superiores a 0,87 , lo cual indicaba una concordancia casi perfecta (total en 15 de ellos). De los tres criterios en los que la concordancia fue moderada, en dos de ellos (las consecuencias y la comprensión y aclaración de dudas) los valores del kappa estaban en el punto medio del intervalo $(0,41-0,60)$ y en el tercero (los riesgos típicos) se procedió al cálculo del índice de concordancia general, dado que la prevalencia era extrema, obteniéndose un valor de 96,3\% que indicaba que el criterio era fiable.

El porcentaje de documentos sin epígrafes disminuyó de $25,1 \%$ en la primera evaluación a $7 \%$ en la cuarta, lo que supuso un descenso del 72,1\%, aunque esta cifra osciló entre el $29,9 \%$ del hospital H9 y el $100 \%$ de los 6 hospitales que en la cuarta evaluación no utilizaban ya DCI sin epígrafes. Hay que señalar que el hospital H9, que es el que presentaba la cifra más elevada de documentos defectuosos en relación con este indicador, había conseguido eliminarlos en la tercera evaluación.

El promedio de defectos por DCI disminuyó de 7,6 en la primera evaluación a 4,5 en la cuarta, lo que supuso un descenso del $40,8 \%$ Al comparar la situación por hospitales, 8 de ellos presentaban descensos en el promedio de incumplimientos que oscilaron entre el 8,9 del hospital H9 y el $89,7 \%$ del 


\section{Tabla 1}

Comparación de los porcentajes de incumplimiento de los datos sobre fecha y lugar del consentimiento y de los datos administrativos del centro, del profesional médico, del paciente y de su representante legal

\begin{tabular}{|c|c|c|c|c|c|c|}
\hline Criterio & $\begin{array}{c}\text { Primera } \\
\text { evaluación }\end{array}$ & $\begin{array}{l}\text { Segunda } \\
\text { evaluación } \\
\text { IC } 95 \%\end{array}$ & $\begin{array}{l}\text { Tercera } \\
\text { evaluación } \\
\text { IC } 95 \%\end{array}$ & $\begin{array}{l}\text { Cuarta } \\
\text { evaluación } \\
\text { IC } 95 \%\end{array}$ & $\begin{array}{c}\text { Diferencia } \\
\text { absoluta } \\
\text { cuarta- } \\
\text { primera }\end{array}$ & $\begin{array}{c}\text { Diferencia } \\
\text { relativa } \\
\text { cuarta } \\
\text { primera }\end{array}$ \\
\hline $\begin{array}{l}\mathrm{C} 1 . \\
\text { Nombre del centro sanitario }\end{array}$ & 18,3 & $\begin{array}{c}14,4 \\
12,5-16,3\end{array}$ & $\begin{array}{c}8,2 \\
6,1-10,4\end{array}$ & $\begin{array}{c}1,1^{*} \\
0,2-1,9\end{array}$ & $-17,2$ & $-94,0$ \\
\hline $\begin{array}{l}\text { C2. } \\
\text { Servicio o unidad }\end{array}$ & 18,1 & $\begin{array}{c}11,3 \\
9,5-13,1\end{array}$ & $\begin{array}{c}12,3 \\
10,1-14,5\end{array}$ & $\begin{array}{c}19,3 \\
16,8-21,8\end{array}$ & 1,2 & $\begin{array}{c}\mathrm{Se} \\
\text { incrementa }\end{array}$ \\
\hline $\begin{array}{l}\text { C3. } \\
\text { Datos del médico informante }\end{array}$ & 35,0 & $\begin{array}{c}28,9 \\
25,9-31,8\end{array}$ & $\begin{array}{c}28,0 \\
25,4-30,7\end{array}$ & $\begin{array}{c}13,0^{*} \\
10,8-15,2\end{array}$ & $-22,0$ & $-62,9$ \\
\hline $\begin{array}{l}\text { C4. } \\
\text { Datos del paciente }\end{array}$ & 12,4 & $\begin{array}{c}9,0 \\
6,9-11,1\end{array}$ & $\begin{array}{c}17,7 \\
15,4-20,0\end{array}$ & $\begin{array}{c}19,4^{*} \\
17,3-21,4\end{array}$ & 7,0 & $\begin{array}{c}\mathrm{Se} \\
\text { incrementa }\end{array}$ \\
\hline $\begin{array}{l}\text { C5. } \\
\text { Datos del representante legal }\end{array}$ & 15,9 & $\begin{array}{c}10,4 \\
8,1-12,8\end{array}$ & $\begin{array}{c}8,4 \\
6,2-10,5\end{array}$ & $\begin{array}{c}9,8^{*} \\
7,8-11,8\end{array}$ & $-6,1$ & $-38,4$ \\
\hline $\begin{array}{l}\text { C6. } \\
\text { Fecha y el lugar donde se firma } \\
\text { el consentimiento }\end{array}$ & 40,2 & $\begin{array}{c}25,0 \\
22,8-27,1\end{array}$ & $\begin{array}{c}21,1 \\
18,3-23,9\end{array}$ & $\begin{array}{c}20,1^{*} \\
17,9-22,4\end{array}$ & $-20,1$ & $-50,0$ \\
\hline
\end{tabular}

H1. Tan sólo el hospital H3 tuvo un ligero incremento en el promedio de defectos por DCI, tras haber conseguido mejorar su resultado en este indicador en la tercera evaluación. La razón de variación entre hospitales del promedio de defectos por DCI en la cuarta evaluación fue de 8,33 , es decir, el centro con el valor máximo (el H3) presentaba un número de incumplimientos más de 8 veces superior al hospital que tenía la frecuencia mínima (el H1).

En la tablas 1, 2 y 3 aparece la evolución de los porcentajes de incumplimiento de los criterios y la diferencia relativa entre la cuarta y la primera evaluación, para el total de los hospitales valorados, desagregando por tipo de criterio. De los 19 criterios valorados 16 presentaron disminuciones estadísticamente significativas en el porcentaje de defectos, con respecto a la primera evaluación. Los descensos se produjeron en la práctica totalidad de los criterios de los grupos dos y tres, relacionados con la comprensión del consentimiento, posibilidad de revocación, autori- zación para la realización del procedimiento, entrega de copia y datos del procedimiento a realizar, con la excepción de la recogida del nombre del procedimiento, donde no se obtuvieron variaciones estadísticamente significativas (tablas 2 y 3 ).

En el primer grupo de criterios, relacionados con la recogida de los datos sobre fecha y lugar del consentimiento y de los datos administrativos del centro, del médico, del paciente y del representante legal (tabla 1), se encontró el único criterio del total de los 19 valorados (la existencia de espacios para la recogida de los datos del paciente) en el que se produjo un incremento estadísticamente significativo en el porcentaje de defectos, tras haberse conseguido una reducción de los mismos en la segunda evaluación. En cuanto al resto de criterios de este grupo, en 4 se obtuvieron mejoras estadísticamente significativas y en 1 (la recogida del servicio o unidad) no se produjeron diferencias.

En la figura 1 se observa la mejora total 


\begin{tabular}{|c|c|c|c|c|c|c|}
\hline \multicolumn{7}{|c|}{\begin{tabular}{|c|} 
Tabla 2 \\
Comparación de los porcentajes de incumplimiento de los datos del procedimiento a \\
realizar
\end{tabular}} \\
\hline Criterio & $\begin{array}{c}\text { Primera } \\
\text { evaluación }\end{array}$ & $\begin{array}{l}\text { Segunda } \\
\text { evaluación } \\
\text { IC } 95 \%\end{array}$ & $\begin{array}{c}\text { Tercera } \\
\text { evaluación } \\
\text { IC } 95 \%\end{array}$ & $\begin{array}{l}\text { Cuarta } \\
\text { evaluación } \\
\text { IC } 95 \%\end{array}$ & $\begin{array}{l}\text { Diferencia } \\
\text { absoluta } \\
\text { cuarta- } \\
\text { primera }\end{array}$ & $\begin{array}{l}\text { Diferencia } \\
\text { relativa } \\
\text { cuarta- } \\
\text { primera }\end{array}$ \\
\hline $\begin{array}{l}\text { C7. } \\
\text { Nombre del procedimiento a realizar }\end{array}$ & 3,4 & $\begin{array}{c}3,9 \\
2,2-5,5\end{array}$ & $\begin{array}{c}2,7 \\
1,4-4,1\end{array}$ & $\begin{array}{c}3,5 \\
1,9-5,1\end{array}$ & 0,1 & $\begin{array}{c}\mathrm{Se} \\
\text { incrementa }\end{array}$ \\
\hline $\begin{array}{l}\text { C8. } \\
\text { Naturaleza y descripción } \\
\text { del procedimiento } \\
\end{array}$ & 38,5 & $\begin{array}{c}28,6 \\
25,1-32,0\end{array}$ & $\begin{array}{c}29,7 \\
26,3-33,1\end{array}$ & $\begin{array}{c}25,6^{*} \\
22,5-28,8\end{array}$ & $-12,9$ & $-33,5$ \\
\hline $\begin{array}{l}\text { C9. } \\
\text { Finalidad del procedimiento }\end{array}$ & 60,0 & $\begin{array}{c}47,3 \\
43,9-50,8\end{array}$ & $\begin{array}{c}54,2 \\
50,6-57,7\end{array}$ & $\begin{array}{c}41,7^{*} \\
38,2-45,1\end{array}$ & $-18,3$ & $-30,5$ \\
\hline \begin{tabular}{|l|} 
C10. \\
Consecuencias relevantes \\
o de importancia \\
\end{tabular} & 91,3 & $\begin{array}{c}82,3 \\
79,3-85,3\end{array}$ & $\begin{array}{c}81,1 \\
78,3-83,8\end{array}$ & $\begin{array}{c}75,2^{*} \\
72,2-78,2\end{array}$ & $-16,1$ & $-17,6$ \\
\hline $\begin{array}{l}\text { C11. } \\
\text { Riesgos probables o típicos }\end{array}$ & 31,5 & $\begin{array}{c}24,8 \\
21,5-28,0\end{array}$ & $\begin{array}{c}23,3 \\
20,2-26,4\end{array}$ & $\begin{array}{c}15,2^{*} \\
12,7-17,7\end{array}$ & $-16,3$ & $-51,7$ \\
\hline \begin{tabular}{|l|} 
C12. \\
Riesgos personalizados \\
\end{tabular} & 33,4 & $\begin{array}{c}22,4 \\
19,2-25,5 \\
\end{array}$ & $\begin{array}{c}20,7 \\
17,5-23,8 \\
\end{array}$ & $\begin{array}{c}22,3^{*} \\
19,8-24,7 \\
\end{array}$ & $-11,1$ & $-33,2$ \\
\hline $\begin{array}{l}\text { C13. } \\
\text { Contraindicaciones }\end{array}$ & 99,2 & $\begin{array}{c}93,0 \\
91,0-95,0\end{array}$ & $\begin{array}{c}89,3 \\
87,1-91,5\end{array}$ & $\begin{array}{c}80,7^{*} \\
78,4-82,9\end{array}$ & $-18,5$ & $-18,6$ \\
\hline \begin{tabular}{|l|} 
C14. \\
Alternativas al procedimiento
\end{tabular} & 62,2 & $\begin{array}{c}43,8 \\
40,4-47,2 \\
\end{array}$ & $\begin{array}{c}36,0 \\
32,5-39,5\end{array}$ & $\begin{array}{c}35,7^{*} \\
32,1-39,3\end{array}$ & $-26,5$ & $-42,6$ \\
\hline
\end{tabular}

\begin{tabular}{|c|c|c|c|c|c|c|}
\hline \multicolumn{7}{|c|}{$\begin{array}{c}\text { Tabla } 3 \\
\text { Comparación de los porcentajes de incumplimiento de los datos sobre comprensión } \\
\text { del consentimiento, posibilidad de revocación, autorización para la realización } \\
\text { y entrega de copia }\end{array}$} \\
\hline Criterio & $\begin{array}{c}\text { Primera } \\
\text { evaluación }\end{array}$ & $\begin{array}{l}\text { Segunda } \\
\text { evaluación } \\
\text { IC } 95 \%\end{array}$ & $\begin{array}{l}\text { Tercera } \\
\text { evaluación } \\
\text { IC 95\% }\end{array}$ & $\begin{array}{l}\text { Cuarta } \\
\text { evaluación } \\
\text { IC } 95 \%\end{array}$ & $\begin{array}{c}\text { Diferencia } \\
\text { absoluta } \\
\text { cuarta- } \\
\text { primera }\end{array}$ & $\begin{array}{c}\text { Diferencia } \\
\text { relativa } \\
\text { cuarta- } \\
\text { primera }\end{array}$ \\
\hline $\begin{array}{l}\text { C15. } \\
\text { Declaración de haber comprendido y aclarado dudas }\end{array}$ & 63,7 & $\begin{array}{c}48,8 \\
45,6-51,9\end{array}$ & $\begin{array}{c}40,7 \\
37,5-43,9\end{array}$ & $\begin{array}{c}30,5^{*} \\
27,8-33,3\end{array}$ & $-33,2$ & $-52,1$ \\
\hline $\begin{array}{l}\text { C16. } \\
\text { Conocimiento posibilidad de revocación }\end{array}$ & 22,0 & $\begin{array}{c}14,3 \\
12,0-16,6\end{array}$ & $\begin{array}{c}6,5 \\
4,5-8,4\end{array}$ & $\begin{array}{c}1,8^{*} \\
0,7-2,8\end{array}$ & $-20,2$ & $-91,8$ \\
\hline $\begin{array}{l}\text { C17. } \\
\text { Espacio para la revocación }\end{array}$ & 18,2 & $\begin{array}{c}14,3 \\
12,4-16,2\end{array}$ & $\begin{array}{c}13,5 \\
11,1-15,9\end{array}$ & $\begin{array}{c}2,9^{*} \\
1,5-4,2\end{array}$ & $-15,3$ & $-84,1$ \\
\hline $\begin{array}{l}\text { C18. } \\
\text { Otorgación del consentimiento }\end{array}$ & 10,7 & $\begin{array}{c}11,2 \\
8,8-13,6\end{array}$ & $\begin{array}{c}10,8 \\
8,8-12,7\end{array}$ & $\begin{array}{c}2,0^{*} \\
1,0-3,0\end{array}$ & $-8,7$ & $-81,3$ \\
\hline $\begin{array}{l}\text { C19. } \\
\text { Entrega de copia }\end{array}$ & 87,6 & $\begin{array}{c}50,0 \\
47,4-52,5\end{array}$ & \begin{tabular}{|c|}
41,1 \\
$39,2-43,0$
\end{tabular} & \begin{tabular}{|c|}
$29,9^{*}$ \\
$27,5-32,4$
\end{tabular} & $-57,7$ & $-65,9$ \\
\hline
\end{tabular}


Figura 1

Diagrama de Pareto antes-después. Total SMS
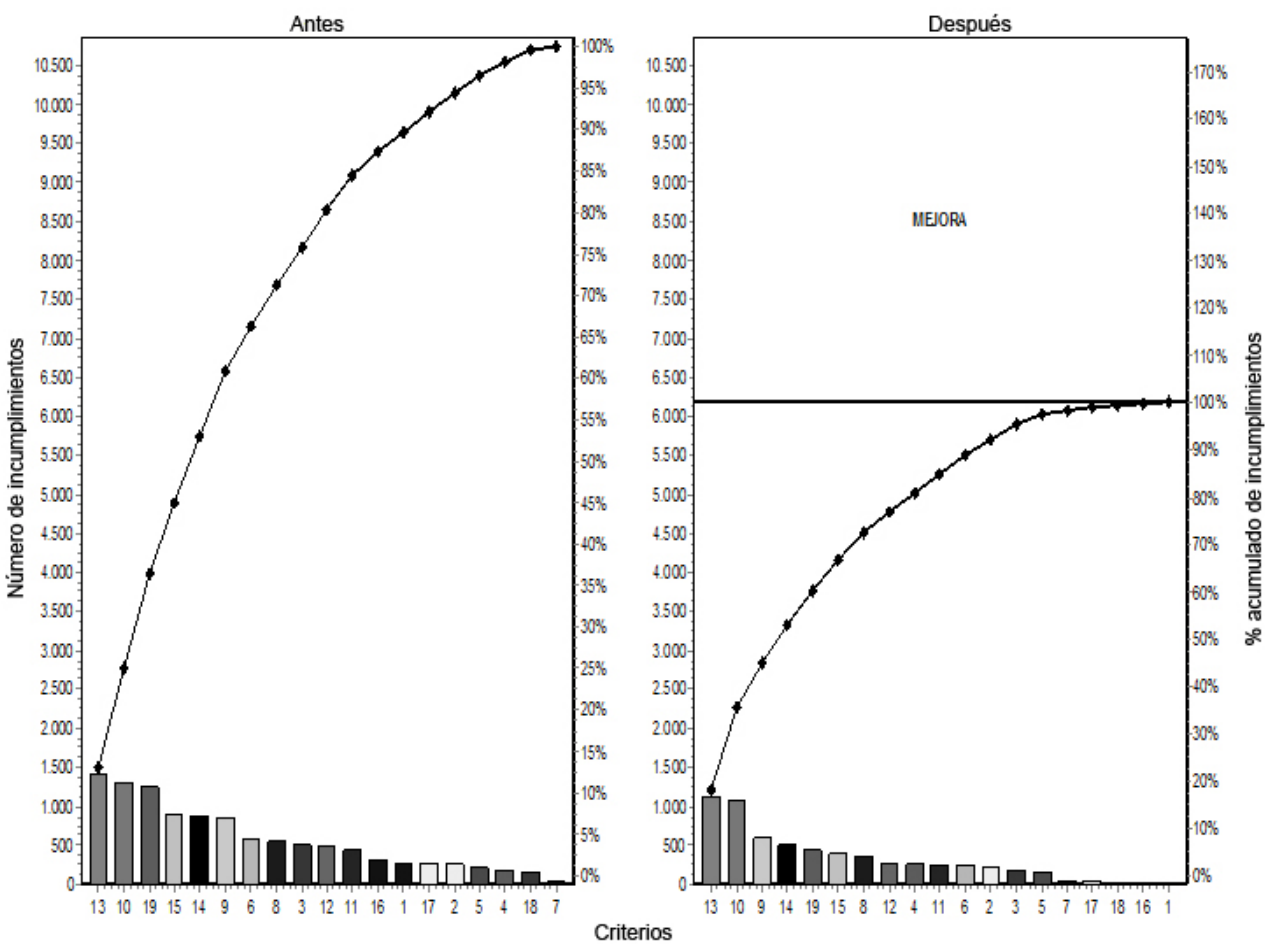

1: Nombre del centro. 2: Servicio o Unidad. 3: Datos del médico. 4: Datos del paciente. 5: Datos del representante legal. 6: Fecha y lugar de dónde se firma. 7: Nombre del procedimiento. 8: Descripción procedimiento. 9: Finalidad. 10: Consecuencias. 11: Riesgos típicos. 12: Riesgos personalizados. 13: Contraindicaciones. 14: Alternativas. 15: Comprensión y dudas. 16: Conocimiento revocación. 17: Espacio para la revocación. 18: Otorgación del consentimiento. 19: Entrega de copia.

conseguida entre la primera y la cuarta evaluación y los criterios que acumularon el mayor margen para la mejora en cada una de las evaluaciones. En la cuarta evaluación 6 de los 19 criterios evaluados acumularon el $66,8 \%$ del total de incumplimientos encontrados para el total del SMS. Ordenados de mayor a menor número de defectos, fueron los criterios relacionados con la presencia en los DCI de información sobre contraindicaciones, consecuencias, finalidad del procedimiento, alternativas al mismo, existencia de alguna expresión relacionada con la entrega de copia del DCI al paciente y de su declaración de haber comprendido la información y de haber aclarado todas las dudas planteadas.

\section{DISCUSIÓN}

Tras la introducción de las medidas correctoras, el número de defectos disminuyó a casi la mitad, con descensos significativos en 16 de los 19 criterios evaluados.

Los ciclos de evaluación y mejora de la calidad son estudios cuasi-experimentales sin grupo control y tienen, por lo tanto, las ventajas e inconvenientes de los mismos ${ }^{12}$. Entre los inconvenientes cabe señalar el que no se puede asegurar que los cambios aparecidos sean debidos a la propia intervención, dado que pueden deberse también a otras intervenciones o aspectos no controlados. El alto porcentaje de mejora obtenido en este trabajo parece confirmar la efectividad de las medidas correctoras 
puestas en marcha. Sin embargo, hay que considerar las numerosas ventajas de los estudios cuasi-experimentales, con respecto a los experimentales puros, como son el menor número de obstáculos para su realización, su menor coste y el que a veces este tipo de diseños son la única alternativa, dado que no parece ético aplicar las mejoras sólo a un grupo y dejar sin ellas a otro para que actúe de control.

Se evaluó la calidad formal de los documentos y no si su contenido era adecuado o no. De todos modos, la evaluación de la calidad formal permite valorar la presencia de todos los apartados de información que la bibliografía sobre el tema establece (validez de contenido), lo cual es básico para que puedan cumplir con sus funciones de orientar el proceso de información y de debate sobre la medida diagnóstica o terapéutica propuesta y de proteger legalmente al médico ${ }^{13}$.

El proceso seguido para la elaboración de los criterios utilizados en este estudio ${ }^{8}$, así como los niveles de fiabilidad alcanzados, los convierte en una herramienta de gran utilidad, tanto para conocer el nivel de calidad de los DCI utilizados actualmente como para valorar y monitorizar el impacto de las medidas correctoras.

Las mejoras no se dieron por igual a todos los centros, dado que en uno de ellos (el H3) se produjo un incremento del porcentaje de defectos y persiste una alta variabilidad entre hospitales en el promedio de incumplimientos por DCI. Es probable que esta variabilidad exista también dentro del hospital pero esta hipótesis no ha podido ser objetivada, dado el alto porcentaje de defectos que presentan algunos centros en relación con la presencia del servicio o unidad a la que pertenece el consentimiento.

El porcentaje de DCI sin epígrafes se reduce también de forma importante en todos los hospitales. Los formularios que utilizan un formato de discusión estructurada pueden facilitar el dialogo médico-paciente ${ }^{14}$.

Si bien se encuentran estudios publicados sobre evaluación y mejora de la calidad del consentimiento informado ${ }^{15-18}$, en ninguno de ellos se realizó un ciclo completo de evaluación y mejora de la calidad formal de los DCI hospitalarios previa a su utilización en pacientes concretos.

Entre los criterios con mayor margen para la mejora tenemos, junto a la entrega de copia del DCI, los relacionados con la capacidad de elección y el grado de comprensión del paciente, en concreto, la información sobre las contraindicaciones, consecuencias relevantes, alternativas, comprensión y aclaración de dudas y finalidad. Estos mismos criterios ocupan los seis primeros lugares en la primera evaluación, pero con distinto orden, dado que todos los criterios relacionados con los datos del procedimiento a realizar escalan posiciones ${ }^{8}$.

Como resumen de todo lo anterior se puede concluir que se están utilizando documentos con problemas de calidad, lo cual es un factor limitante para que los pacientes puedan ejercer el principio de autonomía, es decir, el derecho a decidir libremente, después de recibir la información adecuada entre las opciones clínicas disponibles. Debe tenerse en cuenta además que en los últimos años la mayor parte de procesos judiciales contra médicos se basan en defectos en el proceso de información ${ }^{19,20}$.

Las mejoras alcanzadas con la intervención pueden ayudar a mitigar los problemas y a mejorar la calidad de la interacción médicopaciente y la toma de decisiones compartidas, dado que algunos estudios demuestran que proporcionar información escrita al paciente por medio de DCI de calidad, produce una mejora significativa en la comprensión y el recuerdo

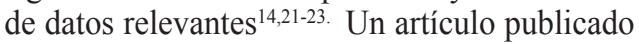
en Estados Unidos señala que el DCI es tan importante, que muchos abogados no aceptarán un caso relacionado con el consentimiento si ven un DCI válido como parte de la evidencia ${ }^{24}$.

En España, una sentencia de 2014 del Tribunal Superior de Justicia de Murcia condenó al Servicio Murciano de Salud al pago de una indemnización de 12.000 euros por las deficiencias detectadas en los DCI, a pesar de que no se había producido una defectuosa práctica 
quirúrgica ni asistencial. El tribunal consideró que los DCI eran claramente insuficientes $\mathrm{y}$ "que se produjo un daño moral a la paciente al privarle de su derecho a decidir y asumir los riesgos y complicaciones inherentes a las intervenciones a las que se sometió, ya que tales deficiencias constituyen en todo caso una mala praxis ad hoc"25. Dado que la reclamación patrimonial se interpuso en 2009, coincidiendo con el inicio de los trabajos de evaluación de estos documentos, una hipótesis a valorar en el futuro es la del posible ahorro económico que la mejora de la calidad los DCI puede suponer para el Servicio Regional de Salud.

Como aún queda margen para la mejora, se debe insistir en la implantación de las medidas correctoras haciendo especial hincapié en los aspectos con mayor número de incumplimientos $\mathrm{y}$, sobre todo, en los criterios relacionados con la presencia en los DCI de la naturaleza del procedimiento sanitario a emplear, sus riesgos, sus beneficios y las alternativas, que son considerados fundamentales por los expertos en este campo ${ }^{14}$.

La utilización en todos los casos del modelo de consentimiento informado que fue aprobado por el grupo de trabajo, hubiera permitido reducir a cero los incumplimientos encontrados en la mayor parte del resto de los criterios (los riesgos personalizados dentro del grupo de datos del procedimiento a realizar y el total de criterios de los otros dos grupos). La dificultad de un proyecto de trabajo como el realizado, en el que han participado a lo largo de varios años los equipos directivos y profesionales de todos los hospitales de un Servicio Regional de Salud, ha podido influir en que este resultado no haya sido alcanzado.

Se debe valorar la posibilidad de diseñar DCI corporativos, tal como han hecho otras Comunidades Autónomas ${ }^{26,27}$, pero aunque algunos aspectos del consentimiento son universales otros están sujetos a discusión y no está claro que todos los centros deban disponer de formularios comunes ${ }^{28}$. No existe unanimidad, por ejemplo, acerca del tipo de alternativas que deben ser ofrecidas al paciente (las que realiza el centro, las incluidas en la cartera de servicios del organismo al que pertenece el mismo o todas las existentes a nivel internacional). Se opte por documentos corporativos o específicos de cada centro, lo importante es que sean de calidad y, para ello, se debe implicar a los profesionales en la evaluación, mejora y actualización periódica de los mismos.

Si el punto de partida es, como en este trabajo, la mejora de los DCI a nivel local, la creación de una base de datos con todos los documentos utilizados por los centros, clasificados por hospital, puede facilitar la posterior elaboración de DCI corporativos.

La utilización de los DCI en una plataforma multimedia, que permita realizar una estimación personalizada de los riesgos, puede contribuir a disminuir la variabilidad en el proceso de comunicación y a mejorar el proceso de toma de decisiones compartidas ${ }^{16,29}$.

\section{BIBLIOGRAFÍA}

1. Sainz A, Quintana O, Sánchez Caro J. La información médica: el consentimiento informado. Fundamentos éticos y legales. Rev Calid Asist. 1994;2:68-71.

2. Simón Lorda P, Concheiro Carro L. El consentimiento informado: teoría y práctica (I). Med Clin (Barc). 1993; 100:659-663.

3. Negro Alvarez JM, Martínez Arrieta F. El consentimiento informado en alergología. Rev Esp Alergol Inmunol Clin. 1995;10:69-77.

4. Boletín Oficial de Estado. Orden de 7 de julio de 1972 por la que se aprueba el Reglamento General para el Régimen, Gobierno y Servicio de las Instituciones Sanitarias de la Seguridad Social. BOE núm 172, 19/7/1972.

5. Boletín Oficial del Estado. Ley 14/1986, de 25 de abril, General de Sanidad. BOE núm 102, 29/4/1986.

6. Boletín Oficial del Estado. Ley 41/2002, de 14 de noviembre, básica reguladora de la autonomía del paciente y de derechos y obligaciones en materia de información y documentación clínica. BOE núm 274, 15/11/2002.

7. Ezeome ER, Chuke PI, Ezeome IV. Contents and readability of currently used surgical/procedure informed consent forms in Nigerian tertiary health institutions. Niger J Clin Pract. 2011;14:311-7. 
8. Calle-Urra JE, Parra-Hidalgo P, Saturno-Hernández PJ, Martínez-Martínez MJ, Navarro-Moya FJ. Evaluación de la calidad formal de los documentos de consentimiento informado en 9 hospitales. Rev Calid Asist. 2013;28:234243.

9. Saturno PJ. Reevaluación, análisis y presentación de resultados comparativos de dos evaluaciones. En: Evaluación y mejora de la calidad en servicios de salud. Herramientas para la realización de ciclos de mejora en gestión de la calidad. Murcia: Consejería de Sanidad y Consumo de la Región de Murcia; 2010.p.183-212.

10. Molina Arias M. El significado de los intervalos de confianza. Rev Pediatr Aten Primaria. 2013;15:91-4.

11. Saturno PJ. Análisis y presentación de los datos de una evaluación. En: Evaluación y mejora de la calidad en servicios de salud. Herramientas para la realización de ciclos de mejora en gestión de la calidad. Murcia: Consejería de Sanidad y Consumo de la Región de Murcia; 2010.p.139168.

12. Molina Arias M, Ochoa Sangrador C. Esayo clínico (I). Tipos. Estudios cuasiexperimentales. Evid Pediatr. 2014;10:52.

13. Simón-Lorda P, Barrio-Cantalejo IM, Concheiro-Carro L. Legibilidad de los formularios escritos de consentimiento informado. Med Clin (Barc). 1997;107:524-29.

14. Bottrell MM, Alpert H, Fischbach RL, Emanuel LL. Hospital informed consent for procedure forms: facilitating quality patient-physician interaction. Arch Surg. 2000;135:26-33.

15. Del Pozo P, Garcia JA, Escribano M, Soria V, Campillo-Soto A, Aguayo-Albasini JL. Evaluación y mejora del manejo del consentimiento informado en urgencias. Rev Calid Asist. 2009;24:222-7.

16. Arnold SV, Decker C, Ahmad H, Olabiyi O, Mundluru $\mathrm{S}$, Reid KJ et als. Converting the informed consent from a perfunctory process to an evidence-based foundation for patient decision making. Circ Cardiovasc Qual Outcomes. 2008;1:21-8.

17. Baños Madrid R, Ramón Esparza T, Serrano Jiménez A, Alajarín Cervera M, Alberca de las Parras F, Molina Martínez J. Evaluación y mejora en la información de los pacientes que se realizan una endoscopia. An Med Interna (Madrid). 2005;9:413-18.

18. González Valverde FM, Gómez Ramos MJ, Méndez Martínez M, Rodenas Moncada FJ, Candel Arenas MF, Albarracín Marín-Blázquez A. Evaluación y mejora del cumplimiento de la normativa sobre consentimiento informado para la transfusión de hemoderivados en un servicio de cirugía general. Rev Calid Asist. 2008;23:142-3.
19. Hospedales-Salomó J, Lloret-Cano MD, BellmuntMontoya S, González-Cañas E, Gonzalo-Villanueva B, Solanich-Valldaura T, et al. ¿Son válidos los documentos de consentimiento informado que utilizamos en cirugía vascular?. Angiología. 2005;57:487-95.

20. Bruguera M, Viger M, Bruguera R, Benet J, Arimany J. Reclamaciones por presunta negligencia relacionadas con endoscopia digestiva. Análisis de una casuística de 22 años. Gastroenterol Hepatol. 2011;34:248-53.

21. Layton S, Korsen J. Informed consent in oral and maxillofacial surgery: a study of the value of written warnings. Br J Oral Maxillofac Surg.1994;32:34-36.

22. Hong P, Makdessian AS, Ellis DA, Taylor SM. Informed consent in rhinoplasty: prospective randomized study of risk recall in patients who are given written disclosure of risks versus traditional oral discussion groups. J Otolaryngol Head Neck Surg.2009;38:369-74.

23. Vergès M, Leclercq F, Davy J-M, Piot C, Gervasoni R, Pasquie JL et al. Les patients bénéficiant d'une coronarographie sont-ils bien informés?. Évaluation de l'efficacité de l'information écrite. Étude prospective unicentrique. Ann Cardiol Angeiol. 2011;60:77-86.

24. Johnson LJ. Malpractice consult. Proper consent forms can be good evidence. Med Econ. 2010 Nov 5;87(21):78.

25. Tribunal Superior de Justicia de Murcia (Sala de lo Contencioso Administrativo) [versión electrónica-base de datos Lefebvre-El Derecho]. Sentencia 589/2014, de 4 de julio de 2014 [consultado 3 marzo 2015].

26. Servicio Andaluz de Salud. Sevilla: Junta de Andalucía; [consultado 30 Ene 2012]. Información clínica para pacientes: Catálogo de formularios de Consentimiento Informado escrito. Disponible en: http://www.juntadeandalucia.es/servicioandaluzdesalud/principal/documentosacc. asp?pagina=gr_actualidad1_b10

27. Portal de la Conselleria de Sanitat. Valencia: Conselleria de Sanitat de la Generalitat Valenciana; [consultado 30 Ene 2012]. Guía de Documentos de Consentimiento Informado. Disponible en: http://www.san.gva.es/comun/ciud/guicastellano.html

28. Kopacova M, Bures J. Informed consent for digestive endoscopy. WJGE. 2012;4:227-30.

29. Terranova G, Ferro M, Carpeggiani C, Recchia V, Braga L, Semelka RC, et al. Low quality and lack of clarity of current informed consent forms in cardiology: how to improve them. JACC Cardiovasc Imaging. 2012;5:649-55. 


\begin{tabular}{|c|c|}
\hline \multicolumn{2}{|c|}{$\begin{array}{c}\text { Anexo } 1 \\
\text { Criterios de calidad utilizados para la evaluación de los documentos de consentimiento } \\
\text { informado de los hospitales generales del Servicio Murciano de Salud }\end{array}$} \\
\hline CRITERIO & ACLARACIONES Y EXCEPCIONES \\
\hline 1. Debe figurar el nombre del centro sanitario & $\begin{array}{l}\text { Se considerará también cumplimiento si aparece el nombre del } \\
\text { área de salud. }\end{array}$ \\
\hline $\begin{array}{l}\text { 2. Debe estar identificado el Servicio o Unidad donde se está } \\
\text { utilizando el documento de consentimiento informado }\end{array}$ & $\begin{array}{l}\text { También se considerará cumplimiento cuando aparezca un espacio } \\
\text { en blanco para anotar el nombre del servicio. }\end{array}$ \\
\hline $\begin{array}{l}\text { 3. Deben contener espacios para anotar el nombre, apellidos, } \\
\text { número de colegiado y firma del médico informante }\end{array}$ & $\begin{array}{l}\text { En relación al número de colegiado, se considerará también cum- } \\
\text { plimiento si aparece, en lugar de este, el código identificativo que } \\
\text { haya sido asignado, por parte de la autoridad sanitaria, al profe- } \\
\text { sional informante. }\end{array}$ \\
\hline $\begin{array}{l}\text { 4. Debe contener espacios para anotar el nombre, apellidos, número } \\
\text { de DNI y firma del paciente al que se va a realizar el procedimiento }\end{array}$ & Excepción : pacientes pediátricos \\
\hline \multicolumn{2}{|l|}{$\begin{array}{l}\text { 5. Debe contener espacios para anotar el nombre, apellidos, } \\
\text { número de DNI y firma del representante legal, familiar o persona } \\
\text { vinculada de hecho que ejerce la representación del paciente }\end{array}$} \\
\hline \multirow{2}{*}{\multicolumn{2}{|c|}{$\begin{array}{l}\text { 6. Debe contener espacios para anotar la fecha y el lugar donde se } \\
\text { firma el consentimiento informado } \\
\text { 7. El nombre del procedimiento a realizar debe aparecer claramente } \\
\text { identificado*. }\end{array}$}} \\
\hline & \\
\hline $\begin{array}{l}\text { 8. Debe aparecer la naturaleza y descripción del procedimiento a } \\
\text { realizar*. }\end{array}$ & $\begin{array}{l}\text { Deberá figurar en que consiste el procedimiento, es decir, que es } \\
\text { lo que se va a hacer. Se considerará cumplimiento cuando aparece } \\
\text { recogido en un apartado específico con el nombre "Descripción } \\
\text { del procedimiento" "En que consiste", "Que le vamos a hacer", } \\
\text { "Cómo se realiza", "Modo de realización" o equivalente. } \\
\text { También será cumplimiento cuando aparezcan en el mismo epí- } \\
\text { grafe la descripción y la finalidad y en el contenido se expliciten } \\
\text { ambos. }\end{array}$ \\
\hline 9. Debe aparecer la finalidad del procedimiento*. & $\begin{array}{l}\text { Para que sirve, que objetivos persigue o que beneficios se esperan } \\
\text { obtener. Debe aparecer recogido en un apartado específico con el } \\
\text { nombre "Finalidad del procedimiento", "Para que sirve", "Para } \\
\text { que se hace", "Beneficios" o equivalente. } \\
\text { También será cumplimiento cuando aparezcan en el mismo epí- } \\
\text { grafe la descripción y la finalidad y en el contenido se expliciten } \\
\text { ambos. }\end{array}$ \\
\hline 10. Deben aparecer las consecuencias relevantes o de importancia*. & $\begin{array}{l}\text { Son aquellas que aparecen con seguridad, en todos los casos, tras } \\
\text { la realización del procedimiento. Deben aparecer en un apartado } \\
\text { específico con el nombre "Consecuencias" o equivalente. En el } \\
\text { caso de que el procedimiento o técnica no tenga este tipo de conse- } \\
\text { cuencias, se deberá señalar este aspecto en este apartado. }\end{array}$ \\
\hline $\begin{array}{l}\text { 11. Deben figurar los riesgos probables en condiciones normales o } \\
\text { riesgos típicos*. }\end{array}$ & $\begin{array}{l}\text { Deben aparecer en un apartado específico con el nombre "Ries- } \\
\text { gos probables", "Riesgos en condiciones normales", "Riesgos } \\
\text { Típicos" "Riesgos", "Efectos secundarios", "Complicaciones" o } \\
\text { equivalente. }\end{array}$ \\
\hline $\begin{array}{l}\text { 12. Debe contener espacios para anotar los riesgos personalizados } \\
\text { de importancia }\end{array}$ & $\begin{array}{l}\text { Son aquellos relacionados con las circunstancias personales o pro- } \\
\text { fesionales del paciente. Deben aparecer en un apartado especíico } \\
\text { con el nombre "Riesgos personalizados", "Riesgos que se añaden } \\
\text { en su caso" o equivalente. }\end{array}$ \\
\hline 13. Deben aparecer las contraindicaciones*. & $\begin{array}{l}\text { Deben aparecer en un apartado específico con el nombre "Contra- } \\
\text { indicaciones" o equivalente. En el caso de que el procedimiento o } \\
\text { técnica no tenga contraindicaciones, se deberá señalar este aspecto } \\
\text { en este apartado. }\end{array}$ \\
\hline 14. Deben aparecer las alternativas al procedimiento*. & $\begin{array}{l}\text { Deben aparecer en un apartado específico con el nombre "Alter- } \\
\text { nativas al procedimiento", "Opciones", "Procedimientos alternati- } \\
\text { vos" o equivalente. Se deberá enumerar claramente la relación de } \\
\text { alternativas de las que se dispone o indicar en caso contrario que } \\
\text { No existen alternativas. }\end{array}$ \\
\hline
\end{tabular}




\begin{tabular}{|c|c|}
\hline \multicolumn{2}{|c|}{$\begin{array}{l}\text { Anexo } 1 \\
\text { continuación }\end{array}$} \\
\hline CRITERIO & ACLARACIONES Y EXCEPCIONES \\
\hline $\begin{array}{l}\text { 15. Debe figurar la declaración por parte del paciente de haber } \\
\text { comprendido adecuadamente la información y de haber aclarado } \\
\text { todas las dudas planteadas }\end{array}$ & $\begin{array}{l}\text { En cuanto a la declaración de haber comprendido, se considerará } \\
\text { correcto cuando especifica que el paciente "ha comprendido ade- } \\
\text { cuadamente". Sin embargo, no será cumplimiento cuando aparece } \\
\text { que "ha sido informado" o expresiones similares. } \\
\text { Por lo que respecta a la aclaración de dudas, se considerará ade- } \\
\text { cuado cuando aparece que el paciente "ha aclarado o tenido la } \\
\text { oportunidad de aclarar todas las dudas planteadas". Sin embargo, } \\
\text { no será cumplimiento cuando aparece que "ha podido plantear to- } \\
\text { das las dudas" o expresiones similares. }\end{array}$ \\
\hline \multicolumn{2}{|c|}{$\begin{array}{l}\text { 16. Debe figurar la declaración de que conoce que el consentimien- } \\
\text { to puede ser revocado en cualquier momento, sin expresión de la } \\
\text { causa de revocación }\end{array}$} \\
\hline $\begin{array}{l}\text { 17. Debe aparecer un espacio para la revocación del consentimien- } \\
\text { to en el caso de que el paciente lo considere necesario }\end{array}$ & $\begin{array}{l}\text { Debe aparecer en un apartado específico con el nombre "Revoca- } \\
\text { ción del consentimiento" o equivalente. }\end{array}$ \\
\hline $\begin{array}{l}\text { 18. Debe figurar la expresión del paciente o de su representante } \\
\text { legal de que da su consentimiento para someterse al procedimiento }\end{array}$ & $\begin{array}{l}\text { Esto suele aparecer con la expresión "DOY MI CONSENTIMIENTO", } \\
\text { "CONSIENTO", "AUTORIZO" o equivalente. }\end{array}$ \\
\hline $\begin{array}{l}\text { 19. Debe constar en el documento que al paciente se le ha hecho } \\
\text { entrega de una copia del mismo }\end{array}$ & \\
\hline
\end{tabular}

